\title{
Students' perceptions of the educational environment at King Abdulaziz University Faculty of Dentistry (KAUFD): a cross sectional study
}

Heba J. Sabbagh ${ }^{1 *}$ (D), Hanin A. Bakhaider ${ }^{1}$, Hesham M. Abokhashabah² and Mohammed U. Bader ${ }^{2}$

\begin{abstract}
Background: Prior research studies have found that dental students' educational environment has an impact on their academic achievement. Therefore, the aim of this cross-sectional study was to assess dental students' perceptions of the educational environment at King Abdulaziz University Faculty of Dentistry (KAUFD) in Saudi Arabia.

Methods: Second-, third-, and fourth-year dental students at KAUFD, responded to the Dundee Ready Education Environment Measure (DREEM) in October 2017. It consists of five subscales: students' perceptions of learning, students' perceptions of teachers, students' academic self-perceptions, students' perceptions of the atmosphere, and students' social self-perceptions. The overall mean value was calculated.

Results: A total of 217 dental students responded to the questionnaire (92 males, 125 females); the response rate was $43.40 \%$. The overall mean DREEM score was 125 , which is considered "more positive than negative." The mean DREEM score was higher for females (128.73) than for males (120.13). Third-year students (137.99) obtained higher mean scores compared to fourth-year (121.42) and fifth-year students (115.94).

Conclusions: Dental students' perceptions of the educational environment at KAUFD support the findings of national and international studies. Students in the preclinical dental academic year (third year) obtained the highest DREEM score, when compared to those who belonged to the clinical academic years. Therefore, a personal development program and good support systems must be emphasized for clinical-year students.
\end{abstract}

Keywords: Educational environment, Saudi Arabia, DREEM, Dental students

\section{Background}

Educational environment is a key factor that influences medical students' education, curriculum satisfaction, learning outcomes, and professional development [1]. Accordingly, improving the educational environment could help establish high-quality university education

\footnotetext{
* Correspondence: hsabbagh@kau.edu.sa

${ }^{1}$ Department of Pediatric Dentistry, King Abdulaziz University, Jeddah, Saudi Arabia

Full list of author information is available at the end of the article
}

and promote students' future success $[2,3]$. It is also an effective strategy to measure the efficiency of the university curriculum and student achievement [1]. The Dundee Ready Education Environment Measure (DREEM) was constructed and validated as a universal diagnostic inventory that can assess educational environment. It was originally validated by a Delphi panel that consisted of approximately 100 medical and health educators from several countries [4].

C C The Author(s). 2020 Open Access This article is licensed under a Creative Commons Attribution 4.0 International License, which permits use, sharing, adaptation, distribution and reproduction in any medium or format, as long as you give appropriate credit to the original author(s) and the source, provide a link to the Creative Commons licence, and indicate if changes were made. The images or other third party material in this article are included in the article's Creative Commons licence, unless indicated otherwise in a credit line to the material. If material is not included in the article's Creative Commons licence and your intended use is not permitted by statutory regulation or exceeds the permitted use, you will need to obtain permission directly from the copyright holder. To view a copy of this licence, visit http://creativecommons.org/licenses/by/4.0/. The Creative Commons Public Domain Dedication waiver (http://creativecommons.org/publicdomain/zero/1.0/) applies to the data made available in this article, unless otherwise stated in a credit line to the data. 
King Abdulaziz University Faculty of Dentistry (KAUFD) in Saudi Arabia has developed strategic goals that help further its mission, namely the achievement of excellence in teaching, learning, and leadership [5]. Their curriculum is described as a traditional curriculum with elements of case-based learning, distributed over a sixyear period, with a comprehensive care dental clinic initiated in the sixth year and a 12-month internship. In addition, in the second and third year, preclinical students undergo an orientation week that entails a personal development program (PDP) that is structured to include workshops and lectures on personal growth, mental health, educational skills, goal setting, and selfimprovement (including ethics and professionalism) (see Table 1). The PDP was designed to improve the quality of student learning, outcomes, and educational environment. Therefore, the aim of this descriptive analytical cross-sectional study was to assess dental students' perceptions of the educational environment at KAUFD using the DREEM.

\section{Methods}

Ethical approval for this study was obtained from the ethics committee at KAUFD (047-03-18). All third-, fourth-, and fifth-year dental students, enrolled at KAUFD, were included in this study. The inclusion criterion was students who were attending classes and lectures taught by the dental faculty at the dental school. Students who were pursuing the basic-sciences year and second year of the dental program were excluded because their lectures pertained primarily to medical and basic sciences taught by professors of the Science and Medical college. The sixth year of the program is the final year during which students are typically in the clinics of the University Dental Hospital and attend a limited number of lectures in classrooms. They are fully occupied with comprehensive dental care clinics, presentations related to their clinical cases, and community activity [6].

Students were approached in October 2017. The study setting was designed to assure comfort and reliability to students. Students were introduced to the study face-toface in their classrooms at noon, after their morning lectures and during their break time, from $12 \mathrm{pm}$ to $1 \mathrm{pm}$.
They were offered a complete verbal explanation of the research methodology and its privacy. They were informed that the study sought their actual opinion and that "truth" is always constructive. They were then invited to participate and sign a consent form for their agreement. The questionnaire was distributed with codes and included no names. The information was gathered from the students through a self-reported questionnaire to allow their free expression. The questionnaire began with students' demographic data (gender, age, and level of their dental education). To measure student perceptions of the educational environment, the DREEM was used. It consists of five subscales:

- Students' perceptions of learning: 12 items, max score 48, and interpreted as follows: $0-12$, very poor; $13-24$, teaching is viewed negatively; $25-36$, a more positive perception; $37-48$, teaching highly thought of.

- Students' perceptions of teachers: 11 items, max score 44, and interpreted as follows: 0-11, abysmal; 12-22, in need of some retraining; $23-33$, moving in the right direction; and 34-44, model teachers.

- Students' academic self-perceptions: 8 items, max score 32, and interpreted as follows: $0-8$, feeling of total failure; 9-16, many negative aspects; $17-24$, feeling more on the positive side; and 25-32, confident.

- Students' perception of atmosphere: 12 items, max score 48, and interpreted as follows: $0-12$, a terrible environment; $13-24$, there are many issues that need to be changed; $25-36$, a more positive atmosphere; and 37-48, a good feeling overall.

- Students' social self-perceptions: 7 items, max score 28, and interpreted as follows: $0-7$, miserable; 8-14, not a nice place; $15-21$, not very bad: and $22-28$, very good socially.

In addition, each subscale includes items that record responses on a 5-point Likert scale ranging from "strongly disagree" (score $=0)$ to "strongly agree" $($ score $=4)$. However, nine of the questions were negative statements and were conversely scored. Means and standard deviations (SD) were calculated for each

Table 1 Distribution of content strategies across the one-week personal development program and its related DREEM subscale

\begin{tabular}{lll}
\hline Session & Strategy & DREEM subscale \\
\hline Session one & Communication \& Interpersonal Skills & • Student's social self-perceptions \\
Session two & Learning Skills and introduction to courses & • Students' perception of learning \\
Session three & Practicing and applying ethics/Professional behavior & Students' academic self-perception \\
Session four & Personality development & $\cdot$ Students' perception of teachers \\
Session five & Humanistic culture and educational environment & $\cdot$ students' perceptions of atmosphere \\
\hline
\end{tabular}


individual item. Individual item values equal to or greater than 3.5 are indicative of a strong presence of the respective aspect of the educational environment, defined as "especially strong," whereas values that are equal to or less than 2 indicate that the respective aspect requires special attention, defined as "to need particular attention"; mid-range values indicate that the respective aspect of the educational environment offers scope for improvement, defined as "could be improved."

Moreover, the overall mean value was calculated. A total DREEM score between 151 and 200 is considered excellent. A score between 101 and 150 is considered more positive than negative. A score below 151 is considered as "plenty of problems" or very poor [7-9].

Statistical analyses were conducted using the Statistical Package for Social Sciences v 16.0 (SPSS Inc., Chicago, IL, USA). T-test and ANOVA were used to calculate statistically significant differences. The Bonferroni correction was used to counteract for multiple comparisons. Significance level was set to 0.05 .

\section{Results}

Out of a total of 500 third, fourth, and fifth-year dental students, 217 responded to the questionnaire (92 males, 125 females); thus, the response rate was $43.40 \%$ (see Table 2). The Cronbach's alpha analysis for overall DREEM was 0.93, suggesting relatively high internal consistency.

The overall mean DREEM score was 125 , which is considered "more positive than negative." The mean DREEM score was higher for females (128.73) than for males (120.13). Third-year students (137.99) obtained higher mean scores compared to fourth-year (121.42) and fifth-year students (115.94).

In addition, regarding the mean total scores that were computed for each subscale, students' perceptions of learning scored 30.4, interpreted as "a more positive approach"; their perception of teachers scored 23.6, interpreted as "moving in the right direction"; their academic self-perception scored 21.7, interpreted as "feeling more on the positive side"; their perception of atmosphere scored 30.4, interpreted as "a more positive atmosphere"; and their social self-perception scored 19.1, interpreted as "not very bad." Third-year students obtained the highest mean scores for all DREEM subscale items (32.29 for

Table 2 Distribution of the sample according to academic year and sex

\begin{tabular}{lllr}
\hline Level & Male & female & $\boldsymbol{P}$ value \\
\hline 3rd year & $27(29.34)$ & $50(40.0)$ & 0.194 \\
4th year & $33(35.87)$ & $33(26.4)$ & \\
5th year & $32(34.78)$ & $42(33.6)$ & \\
Total & $\mathbf{9 2 ( 1 0 0 )}$ & $\mathbf{1 2 5 ( 1 0 0 )}$ & $\mathbf{2 1 7}$ \\
\hline
\end{tabular}

students' perceptions of learning, 25.97 for students' perceptions of teachers, 23.77 for students' academic selfperceptions, 34.31 for atmosphere perceptions, and 21.65 for students' social self-perceptions) followed by fourth- and fifth-year students (see Table 3).

Moreover, the mean item scores were mostly between 2 and 3.50 across all academic years and genders; these scores were representative of a moderate "could be improved" level. There were statistically significant differences between the three academic years regarding their DREEM item mean scores represented by the ANOVA results (see Table 3) and the Bonferroni correction for multiple comparison (see Supplementary Table 1).

\section{Discussion}

The KAUFD curriculum is oriented toward the mission of achieving excellence in teaching, learning, and leadership [5]. This could not have been established without improving the educational environment of the institute because it is essential for student academic achievement [7]. In this study, the DREEM was used to measure the overall educational environment; we also examined other factors such as academic year and sex that could affect student perception of the educational environment.

In this study, the mean of the composite DREEM scores for the third-, fourth-, and fifth-year dental students was 125 . This value is similar to the means obtained in previous national studies conducted in medical and dental schools: Al-Quaseem $(\mathrm{M}=102),[10] \mathrm{Al}$ Madina $(M=106)$, [11] Riyadh $(M=108.42),[12]$ and Dammam $(M=112.38)$ [13]. In addition, comparable studies carried out in other parts of the world have reported similar or greater variability in mean DREEM scores: Iran (99.60), [14] Trinidad and Tobago (109.90), [15] Nigeria (118), Germany (122.95), [16] the United Kingdom (124), [8] and Nepal (130) [17]. A more recent study that assessed the academic environment of a 4 year inquiry-based dental curriculum in the United Kingdom reported a high mean DREEM score of 143.58 [18]. The different lengths of educational years and type of curriculum could have contributed to the different DREEM outcome.

Regarding gender differences, female students obtained a slightly higher mean DREEM score than male students. Females obtained significantly higher mean scores on items that were related to teaching strategies, teachers, and their social lives in school. This finding is consistent with those of past studies [11, 15, 16].

In addition, the mean DREEM score was significantly higher among third-year students, as compared to fourth- and fifth-year students. This finding is consistent with those of previous studies of medical schools in both Spain [19] and Turkey [20]. Specifically, the researchers found that preclinical-year students had more positive 
Table 3 Means for the 50 items of the DREEM as a function of academic year and sex

\begin{tabular}{|c|c|c|c|c|c|c|c|}
\hline DREEM Items & $\begin{array}{l}\text { Male } \\
\text { Mean } \\
(\mathrm{SD})\end{array}$ & $\begin{array}{l}\text { female } \\
\text { Mean } \\
\text { (SD) }\end{array}$ & $\begin{array}{l}P \\
\text { value }\end{array}$ & $\begin{array}{l}\text { Third } \\
\text { year } \\
\text { Mean } \\
\text { (SD) }\end{array}$ & $\begin{array}{l}\text { Fourth } \\
\text { year } \\
\text { Mean } \\
\text { (SD) }\end{array}$ & $\begin{array}{l}\text { Fifth year } \\
\text { Mean } \\
\text { (SD) }\end{array}$ & $\begin{array}{l}P \\
\text { value }\end{array}$ \\
\hline \multicolumn{8}{|l|}{ I. Student's perceptions of learning (Max 48) } \\
\hline I am encouraged to participate in class & $\begin{array}{l}2.59 \\
(1.187)\end{array}$ & $\begin{array}{l}2.58 \\
(.835)\end{array}$ & 0.983 & $\begin{array}{l}2.70 \\
(1.113)\end{array}$ & $\begin{array}{l}2.58 \\
(0.895)\end{array}$ & $\begin{array}{l}2.47 \\
(0.954)\end{array}$ & 0.372 \\
\hline The teaching concerned to develop my confidence & $\begin{array}{l}2.63 \\
(1.116)\end{array}$ & $\begin{array}{l}2.61 \\
(.932)\end{array}$ & 0.872 & $\begin{array}{l}2.88 \\
(0.973)\end{array}$ & $\begin{array}{l}2.62 \\
(1.004)\end{array}$ & $\begin{array}{l}2.34 \\
(0.997)\end{array}$ & $0.004^{*}$ \\
\hline The teaching encourages me to be active learner & $\begin{array}{l}2.60 \\
(1.110)\end{array}$ & $\begin{array}{l}2.75 \\
(.886)\end{array}$ & 0.257 & $\begin{array}{l}2.95 \\
(1.012)\end{array}$ & $\begin{array}{l}2.70 \\
(0.928)\end{array}$ & $\begin{array}{l}2.41 \\
(0.950)\end{array}$ & $0.003^{*}$ \\
\hline The teaching is well focused & $\begin{array}{l}2.62 \\
(1.098)\end{array}$ & $\begin{array}{l}2.98 \\
(.893)\end{array}$ & $0.009^{*}$ & $\begin{array}{l}3.14 \\
(0.942)\end{array}$ & $\begin{array}{l}2.71 \\
(1.034)\end{array}$ & $\begin{array}{l}2.59 \\
(0.950)\end{array}$ & $0.002^{*}$ \\
\hline The teaching concerned to develop my competence & $\begin{array}{l}2.58 \\
(1.092)\end{array}$ & $\begin{array}{l}2.74 \\
(.941)\end{array}$ & 0.226 & $\begin{array}{l}2.95 \\
(0.916)\end{array}$ & $\begin{array}{l}2.59 \\
(1.007)\end{array}$ & $\begin{array}{l}2.46 \\
(1.049)\end{array}$ & $0.008^{*}$ \\
\hline I am clear about the course learning objectives & $\begin{array}{l}2.58 \\
(1.122)\end{array}$ & $\begin{array}{l}2.78 \\
(1.077)\end{array}$ & 0.186 & $\begin{array}{l}3.03 \\
(1.051)\end{array}$ & $\begin{array}{l}2.74 \\
(0.917)\end{array}$ & $\begin{array}{l}2.30 \\
(1.179)\end{array}$ & $\begin{array}{l}< \\
0.001\end{array}$ \\
\hline The teaching is often stimulating & $\begin{array}{l}2.47 \\
(1.032)\end{array}$ & $\begin{array}{l}2.62 \\
(.811)\end{array}$ & 0.236 & $\begin{array}{l}2.74 \\
(0.979)\end{array}$ & $\begin{array}{l}2.67 \\
(0.829)\end{array}$ & $\begin{array}{l}2.26 \\
(0.845)\end{array}$ & $0.002^{*}$ \\
\hline The teaching time is put to good use & $\begin{array}{l}2.25 \\
(1.210)\end{array}$ & $\begin{array}{l}2.58 \\
(1.049)\end{array}$ & $0.031^{*}$ & $\begin{array}{l}2.81 \\
(1.014)\end{array}$ & $\begin{array}{l}2.36 \\
(1.185)\end{array}$ & $\begin{array}{l}2.14 \\
(1.102)\end{array}$ & $0.001^{*}$ \\
\hline The teaching is student-centered & $\begin{array}{l}2.46 \\
(1.042)\end{array}$ & $\begin{array}{l}2.58 \\
(.854)\end{array}$ & 0.355 & $\begin{array}{l}2.86 \\
(0.884)\end{array}$ & $\begin{array}{l}2.42 \\
(0.993)\end{array}$ & $\begin{array}{l}2.27 \\
(0.849)\end{array}$ & $\begin{array}{l}< \\
0.001 *\end{array}$ \\
\hline Long-term learning is emphasized over the short term & $2.8(0.92)$ & $\begin{array}{l}2.55 \\
(1.17)\end{array}$ & 0.51 & $\begin{array}{l}2.15 \\
(1.18)\end{array}$ & $3.1(0.89)$ & $3.1(1.21)$ & $0.031^{*}$ \\
\hline The teaching is too teacher-centered ${ }^{a}$ & $1.9(0.88)$ & $1.55(1.1)$ & 0.325 & $\begin{array}{l}1.38 \\
(0.96)\end{array}$ & $\begin{array}{l}1.4 \\
(1.001)\end{array}$ & $\begin{array}{l}2.03 \\
(1.18)\end{array}$ & $0.036^{*}$ \\
\hline The teaching overemphasizes factual learning ${ }^{a}$ & $\begin{array}{l}2.21 \\
(0.79)\end{array}$ & $\begin{array}{l}2.68 \\
(0.87)\end{array}$ & 0.096 & $2.7(0.82)$ & $\begin{array}{l}2.55 \\
(0.93)\end{array}$ & $2.8(0.87)$ & $0.033^{*}$ \\
\hline Total & 29.69 & 31.1 & & 32.29 & 30.44 & 29.17 & \\
\hline \multicolumn{8}{|l|}{ II. Student's perceptions of teachers (Max 44): } \\
\hline The teacher providing feedback to student & $\begin{array}{l}2.47 \\
(1.133)\end{array}$ & $\begin{array}{l}2.62 \\
(.965)\end{array}$ & 0.299 & $\begin{array}{l}3.03 \\
(1.000)\end{array}$ & $\begin{array}{l}2.29 \\
(1.049)\end{array}$ & $\begin{array}{l}2.30 \\
(0.903)\end{array}$ & $\begin{array}{l}< \\
0.001 *\end{array}$ \\
\hline The teachers have good communications skills with patients & $2.5(0.97)$ & $\begin{array}{l}2.62 \\
(0.85)\end{array}$ & 0.66 & $2.5(0.79)$ & $2.6(0.84)$ & $2.7(0.97)$ & 0.884 \\
\hline The teachers give clear examples & $\begin{array}{l}2.65 \\
(.966)\end{array}$ & $\begin{array}{l}2.93 \\
(.938)\end{array}$ & 0.041 & $\begin{array}{l}3.14 \\
(0.854)\end{array}$ & $\begin{array}{l}2.73 \\
(0.851)\end{array}$ & $\begin{array}{l}2.53 \\
(1.050)\end{array}$ & $\begin{array}{l}< \\
0.001 *\end{array}$ \\
\hline The teachers prepared for their classes & $\begin{array}{l}2.89 \\
(1.114)\end{array}$ & $\begin{array}{l}3.09 \\
(1.008)\end{array}$ & 0.176 & $\begin{array}{l}3.47 \\
(0.788)\end{array}$ & $\begin{array}{l}2.91 \\
(1.077)\end{array}$ & $\begin{array}{l}2.61 \\
(1.108)\end{array}$ & $\begin{array}{l}< \\
0.001^{*}\end{array}$ \\
\hline The teachers provide constructive criticism & $\begin{array}{l}2.41 \\
(.985)\end{array}$ & $\begin{array}{l}2.74 \\
(.817)\end{array}$ & $0.011^{*}$ & $\begin{array}{l}2.73 \\
(0.883)\end{array}$ & $\begin{array}{l}2.67 \\
(0.883)\end{array}$ & $\begin{array}{l}2.39 \\
(0.919)\end{array}$ & $0.054^{*}$ \\
\hline The students irritate the teachers ${ }^{a}$ & $\begin{array}{l}1.73 \\
(1.017)\end{array}$ & $\begin{array}{l}2.02 \\
(1.085)\end{array}$ & 0.049 & $\begin{array}{l}1.88 \\
(1.051)\end{array}$ & $\begin{array}{l}1.89 \\
(1.191)\end{array}$ & $\begin{array}{l}1.91 \\
(0.968)\end{array}$ & 0.992 \\
\hline The teachers ridicule the students a & $1.8(1.03)$ & $\begin{array}{l}2.05 \\
(1.09)\end{array}$ & 0.5 & $\begin{array}{l}2.23 \\
(1.135)\end{array}$ & $1.7(1.06)$ & $\begin{array}{l}2.07 \\
(0.998)\end{array}$ & 0.12 \\
\hline The teachers get angry in class a & $1.4(0.84)$ & $\begin{array}{l}1.78 \\
(0.94)\end{array}$ & 0.235 & $\begin{array}{l}1.74 \\
(1.04)\end{array}$ & $\begin{array}{l}1.6 \\
(0.814)\end{array}$ & $\begin{array}{l}1.86 \\
(0.915)\end{array}$ & 0.56 \\
\hline The teachers are authoritarian a & $2.1(1.01)$ & $\begin{array}{l}2.08 \\
(0.97)\end{array}$ & 0.9 & $\begin{array}{l}2.13 \\
(0.913)\end{array}$ & $1.9(1.03)$ & $\begin{array}{l}2.21 \\
(0.99)\end{array}$ & 0.45 \\
\hline The professors considered my role-models & $\begin{array}{l}2.53 \\
(1.253)\end{array}$ & $\begin{array}{l}2.78 \\
(1.267)\end{array}$ & 0.132 & $\begin{array}{l}3.12 \\
(1.100)\end{array}$ & $\begin{array}{l}2.59 \\
(1.228)\end{array}$ & $\begin{array}{l}2.28 \\
(1.330)\end{array}$ & $\begin{array}{l}< \\
0.001^{*}\end{array}$ \\
\hline Total & 22.48 & 24.71 & & 25.97 & 22.88 & 22.86 & \\
\hline \multicolumn{8}{|l|}{ III. Student's academic self-perceptions (Max 32) } \\
\hline Memorize all I need & 2.48 & 2.32 & 0.273 & 2.66 & 2.15 & 2.31 & $0.010^{*}$ \\
\hline
\end{tabular}


Table 3 Means for the 50 items of the DREEM as a function of academic year and sex (Continued)

\begin{tabular}{|c|c|c|c|c|c|c|c|}
\hline DREEM Items & $\begin{array}{l}\text { Male } \\
\text { Mean } \\
(\mathrm{SD})\end{array}$ & $\begin{array}{l}\text { female } \\
\text { Mean } \\
\text { (SD) }\end{array}$ & $\begin{array}{l}P \\
\text { value }\end{array}$ & $\begin{array}{l}\text { Third } \\
\text { year } \\
\text { Mean } \\
\text { (SD) }\end{array}$ & $\begin{array}{l}\text { Fourth } \\
\text { year } \\
\text { Mean } \\
\text { (SD) }\end{array}$ & $\begin{array}{l}\text { Fifth year } \\
\text { Mean } \\
\text { (SD) }\end{array}$ & $\begin{array}{l}P \\
\text { value }\end{array}$ \\
\hline & $(1.000)$ & $(1.082)$ & & $(0.954)$ & $(1.056)$ & $(1.084)$ & \\
\hline Much of what I must learn seems relevant to a career in dentistry & $\begin{array}{l}2.41 \\
(1.081)\end{array}$ & $\begin{array}{l}2.94 \\
(.910)\end{array}$ & $\begin{array}{l}< \\
0.001 *\end{array}$ & $\begin{array}{l}3.04 \\
(0.979)\end{array}$ & $\begin{array}{l}2.67 \\
(0.950)\end{array}$ & $\begin{array}{l}2.43 \\
(1.035)\end{array}$ & $0.001^{*}$ \\
\hline I feel I am being well prepared for my profession & $\begin{array}{l}2.50 \\
(1.153)\end{array}$ & $\begin{array}{l}2.90 \\
(.962)\end{array}$ & $0.005^{*}$ & $\begin{array}{l}3.16 \\
(0.947)\end{array}$ & $\begin{array}{l}2.68 \\
(1.055)\end{array}$ & $\begin{array}{l}2.34 \\
(1.037)\end{array}$ & $\begin{array}{l}< \\
0.001 *\end{array}$ \\
\hline Last year's work has been good preparation for this year's work & $\begin{array}{l}2.29 \\
(1.245)\end{array}$ & $\begin{array}{l}2.67 \\
(1.076)\end{array}$ & $0.017^{*}$ & $\begin{array}{l}2.88 \\
(1.026)\end{array}$ & $\begin{array}{l}2.42 \\
(1.266)\end{array}$ & $\begin{array}{l}2.20 \\
(1.110)\end{array}$ & $0.001^{*}$ \\
\hline My problem-solving skills are being developed & $\begin{array}{l}2.67 \\
(1.214)\end{array}$ & $\begin{array}{l}2.95 \\
(.958)\end{array}$ & 0.061 & $\begin{array}{l}3.17 \\
(0.938)\end{array}$ & $\begin{array}{l}2.65 \\
(1.183)\end{array}$ & $\begin{array}{l}2.65 \\
(1.052)\end{array}$ & $0.003^{*}$ \\
\hline I am confident about passing this year & $\begin{array}{l}2.95 \\
(1.142)\end{array}$ & $\begin{array}{l}3.17 \\
(1.098)\end{array}$ & 0.149 & $\begin{array}{l}3.47 \\
(0.912)\end{array}$ & $\begin{array}{l}3.08 \\
(1.057)\end{array}$ & $\begin{array}{l}2.66 \\
(1.231)\end{array}$ & $\begin{array}{l}< \\
0.001^{*}\end{array}$ \\
\hline I have learned a lot about empathy in my profession & $\begin{array}{l}2.73 \\
(1.234)\end{array}$ & $\begin{array}{l}3.02 \\
(1.096)\end{array}$ & $0.055^{*}$ & $\begin{array}{l}2.99 \\
(1.094)\end{array}$ & $\begin{array}{l}3.06 \\
(1.080)\end{array}$ & $\begin{array}{l}2.65 \\
(1.276)\end{array}$ & $0.076^{*}$ \\
\hline $\begin{array}{l}\text { Learning strategies which worked for me before continuing to } \\
\text { work for me now }\end{array}$ & $\begin{array}{l}2.7 \\
(0.675)\end{array}$ & $\begin{array}{l}2.52 \\
(0.97)\end{array}$ & 0.315 & $2.4(1.14)$ & $2.19(1.3)$ & $2.8(0.77)$ & 0.46 \\
\hline Total & 20.82 & 22.49 & & 23.77 & 20.9 & 20.04 & \\
\hline \multicolumn{8}{|l|}{ IV. students' perceptions of atmosphere (Max 48) } \\
\hline Atmosphere during lecture & $\begin{array}{l}2.47 \\
(1.074)\end{array}$ & $\begin{array}{l}2.62 \\
(1.162)\end{array}$ & 0.338 & $\begin{array}{l}3.18 \\
(0.899)\end{array}$ & $\begin{array}{l}2.24 \\
(1.096)\end{array}$ & $\begin{array}{l}2.18 \\
(1.090)\end{array}$ & $\begin{array}{l}< \\
0.001 *\end{array}$ \\
\hline Able to ask what I want & $\begin{array}{l}2.70 \\
(1.146)\end{array}$ & $\begin{array}{l}2.92 \\
(1.052)\end{array}$ & 0.136 & $\begin{array}{l}3.16 \\
(1.089)\end{array}$ & $\begin{array}{l}2.71 \\
(0.924)\end{array}$ & $\begin{array}{l}2.58 \\
(1.170)\end{array}$ & $0.003^{*}$ \\
\hline Socially comfortable in class & $\begin{array}{l}2.83 \\
(1.135)\end{array}$ & $\begin{array}{l}2.99 \\
(1.028)\end{array}$ & 0.262 & $\begin{array}{l}3.29 \\
(0.958)\end{array}$ & $\begin{array}{l}2.91 \\
(1.003)\end{array}$ & $\begin{array}{l}2.55 \\
(1.136)\end{array}$ & $\begin{array}{l}< \\
0.001^{*}\end{array}$ \\
\hline A chance to develop skills & $\begin{array}{l}2.73 \\
(1.080)\end{array}$ & $\begin{array}{l}2.76 \\
(1.095)\end{array}$ & 0.832 & $\begin{array}{l}3.08 \\
(1.133)\end{array}$ & $\begin{array}{l}2.67 \\
(0.950)\end{array}$ & $\begin{array}{l}2.47 \\
(1.076)\end{array}$ & $0.002^{*}$ \\
\hline Atmosphere during tutorials & $\begin{array}{l}2.61 \\
(1.079)\end{array}$ & $\begin{array}{l}2.51 \\
(1.075)\end{array}$ & 0.514 & $\begin{array}{l}2.90 \\
(0.995)\end{array}$ & $\begin{array}{l}2.47 \\
(0.980)\end{array}$ & $\begin{array}{l}2.27 \\
(1.150)\end{array}$ & $0.001^{*}$ \\
\hline The enjoyment outweighs the Stress of studying dentistry & $\begin{array}{l}2.27 \\
(1.351)\end{array}$ & $\begin{array}{l}2.31 \\
(1.174)\end{array}$ & 0.815 & $\begin{array}{l}2.81 \\
(1.124)\end{array}$ & $\begin{array}{l}2.14 \\
(1.239)\end{array}$ & $\begin{array}{l}1.91 \\
(1.218)\end{array}$ & $\begin{array}{l}< \\
0.001 *\end{array}$ \\
\hline Atmosphere motivate me as a learner & $\begin{array}{l}2.33 \\
(1.101)\end{array}$ & $\begin{array}{l}2.54 \\
(1.154)\end{array}$ & 0.162 & $\begin{array}{l}2.99 \\
(1.032)\end{array}$ & $\begin{array}{l}2.32 \\
(0.947)\end{array}$ & $\begin{array}{l}2.01 \\
(1.176)\end{array}$ & $\begin{array}{l}< \\
0.001^{*}\end{array}$ \\
\hline I can concentrate well & $\begin{array}{l}2.50 \\
(1.074)\end{array}$ & $\begin{array}{l}2.62 \\
(.990)\end{array}$ & 0.412 & $\begin{array}{l}2.99 \\
(0.866)\end{array}$ & $\begin{array}{l}2.33 \\
(1.086)\end{array}$ & $\begin{array}{l}2.34 \\
(0.997)\end{array}$ & $\begin{array}{l}< \\
0.001 *\end{array}$ \\
\hline Atmosphere during ward & $\begin{array}{l}2.60 \\
(.984)\end{array}$ & $\begin{array}{l}2.61 \\
(.975)\end{array}$ & 0.940 & $\begin{array}{l}2.88 \\
(0.873)\end{array}$ & $\begin{array}{l}2.47 \\
(0.996)\end{array}$ & $\begin{array}{l}2.43 \\
(1.008)\end{array}$ & $0.007^{*}$ \\
\hline This school is well timetabled & $\begin{array}{l}2.42 \\
(1.277)\end{array}$ & $\begin{array}{l}2.62 \\
(1.037)\end{array}$ & 0.205 & $\begin{array}{l}2.81 \\
(1.064)\end{array}$ & $\begin{array}{l}2.45 \\
(1.205)\end{array}$ & $\begin{array}{l}2.34 \\
(1.138)\end{array}$ & $0.033^{*}$ \\
\hline Experience disappointing & $\begin{array}{l}2.09 \\
(1.315)\end{array}$ & $\begin{array}{l}2.44 \\
(1.153)\end{array}$ & $0.037^{*}$ & $\begin{array}{l}2.78 \\
(1.382)\end{array}$ & $\begin{array}{l}2.08 \\
(1.114)\end{array}$ & $\begin{array}{l}1.97 \\
(1.006)\end{array}$ & $\begin{array}{l}< \\
0.001 *\end{array}$ \\
\hline Cheating is a problem in this school ${ }^{a}$ & $\begin{array}{l}1.39 \\
(1.490)\end{array}$ & $\begin{array}{l}1.86 \\
(1.376)\end{array}$ & $0.017^{*}$ & $\begin{array}{l}1.44 \\
(1.381)\end{array}$ & $\begin{array}{l}1.89 \\
(1.50)\end{array}$ & $\begin{array}{l}1.69 \\
(1.433)\end{array}$ & $0.171^{*}$ \\
\hline Total & 28.94 & 30.8 & & 34.31 & 28.68 & 26.74 & \\
\hline \multicolumn{8}{|l|}{ V. Student's social self-perceptions (Max 28) } \\
\hline I have good friends in this school & $\begin{array}{l}3.11 \\
(1.320)\end{array}$ & $\begin{array}{l}3.30 \\
(1.010)\end{array}$ & 0.222 & $\begin{array}{l}3.47 \\
(0.887)\end{array}$ & $\begin{array}{l}3.41 \\
(1.095)\end{array}$ & $\begin{array}{l}2.80 \\
(1.324)\end{array}$ & $\begin{array}{l}< \\
0.001 *\end{array}$ \\
\hline Good support system who get stressed & $\begin{array}{l}1.98 \\
(1.367)\end{array}$ & $\begin{array}{l}2.18 \\
(1.240)\end{array}$ & 0.249 & $\begin{array}{l}2.64 \\
(1.157)\end{array}$ & $\begin{array}{l}1.79 \\
(1.283)\end{array}$ & $\begin{array}{l}1.81 \\
(1.279)\end{array}$ & $\begin{array}{l}< \\
0.001^{*}\end{array}$ \\
\hline Too tired to enjoy the course ${ }^{a}$ & $1.48(1.2)$ & $\begin{array}{l}1.63 \\
(1.051)\end{array}$ & 0.317 & $\begin{array}{l}1.9 \\
(1.095)\end{array}$ & $\begin{array}{l}1.29 \\
(1.092)\end{array}$ & $\begin{array}{l}1.47 \\
(1.088)\end{array}$ & $0.003^{*}$ \\
\hline Rarely bored on course & 2.04 & 1.92 & 0.414 & 2.09 & 2.02 & 1.81 & 0.273 \\
\hline
\end{tabular}


Table 3 Means for the 50 items of the DREEM as a function of academic year and sex (Continued)

\begin{tabular}{|c|c|c|c|c|c|c|c|}
\hline DREEM Items & $\begin{array}{l}\text { Male } \\
\text { Mean } \\
\text { (SD) }\end{array}$ & $\begin{array}{l}\text { female } \\
\text { Mean } \\
\text { (SD) }\end{array}$ & $\begin{array}{l}P \\
\text { value }\end{array}$ & $\begin{array}{l}\text { Third } \\
\text { year } \\
\text { Mean } \\
\text { (SD) }\end{array}$ & $\begin{array}{l}\text { Fourth } \\
\text { year } \\
\text { Mean } \\
\text { (SD) } \\
\end{array}$ & $\begin{array}{l}\text { Fifth year } \\
\text { Mean } \\
\text { (SD) }\end{array}$ & $\begin{array}{l}P \\
\text { value }\end{array}$ \\
\hline & $(1.109)$ & $(1.090)$ & & $(1.090)$ & $(1.074)$ & $(1.119)$ & \\
\hline My accommodation is pleasant & $\begin{array}{l}2.46 \\
(1.073)\end{array}$ & $\begin{array}{l}2.58 \\
(.909)\end{array}$ & 0.377 & $\begin{array}{l}2.81 \\
(1.052)\end{array}$ & $\begin{array}{l}2.47 \\
(0.964)\end{array}$ & $\begin{array}{l}2.28 \\
(0.852)\end{array}$ & $0.004^{*}$ \\
\hline My social life is good & $\begin{array}{l}2.39 \\
(1.374)\end{array}$ & $\begin{array}{l}2.75 \\
(1.133)\end{array}$ & $0.036^{*}$ & $\begin{array}{l}2.81 \\
(1.148)\end{array}$ & $\begin{array}{l}2.61 \\
(1.402)\end{array}$ & $\begin{array}{l}2.38 \\
(1.190)\end{array}$ & 0.111 \\
\hline I seldom feel lonely & $\begin{array}{l}2.34 \\
(1.122)\end{array}$ & $\begin{array}{l}2.30 \\
(1.157)\end{array}$ & 0.794 & $\begin{array}{l}2.44 \\
(1.106)\end{array}$ & $\begin{array}{l}2.41 \\
(1.176)\end{array}$ & $\begin{array}{l}2.09 \\
(1.125)\end{array}$ & 0.125 \\
\hline I feel belonging to my school & $\begin{array}{l}2.53 \\
(1.471)\end{array}$ & $\begin{array}{l}3.10 \\
(1.260)\end{array}$ & $0.002^{*}$ & $\begin{array}{l}3.49 \\
(0.955)\end{array}$ & $\begin{array}{l}2.52 \\
(1.481)\end{array}$ & $\begin{array}{l}2.49 \\
(1.436)\end{array}$ & $\begin{array}{l}< \\
0.001^{*}\end{array}$ \\
\hline Total & 18.32 & 19.76 & & 21.65 & 18.52 & 17.13 & \\
\hline Mean total score & 120.93 & 128.93 & & 137.99 & 121.42 & 115.94 & \\
\hline
\end{tabular}

Item record responses on a 5-point Likert scale ranging from "strongly disagree" (score $=0)$ to "strongly agree" $($ score $=4)$

${ }^{*} P$ value is significant at 0.05

${ }^{a}$ Questions with negative statement and reversely scored

perceptions of their educational environment than clinical-year students. This finding is also supported by that of a study of medical students in Imam Abdulrahman Bin Faisal University, Dammam, Saudi Arabia [13], where the highest DREEM score was obtained by thirdyear students. The researchers speculated that the recency of enrollment might have augmented student motivation as they were still exploring the educational environment. Similarly, with regard to KAUFD, the finding may be attributable to the PDP that students had attended during the first week of their second and third academic years. Table 1 lists the PDP session strategies and their related DREEM subscale.

There is worldwide agreement that PDPs are important because they equip medical students with the skills they need for their academic studies [21]. Moreover, several studies have reported that, in order to be successful in the medical field, students require not only knowledge and clinical skills but also interpersonal and communication skills [22]. In a previous study, a PDP that was similar to the program conducted in the present study (five sessions for $15 \mathrm{~h}$ across 5 days) was administered to medical and nursing students in India. The students provided feedback, whereby they evaluated the content and outcomes of the program; the researchers found that the students who had attended the PDP showed improvement in their interpersonal and leadership skills, time management skills, emotional stability, and the ability to cope with stress and have a stable social life [22]. This finding might indicate the need for a PDP as part of students' curriculum which would be repeated annually. Another recommendation is that teachers should play a more active role in the maintenance of student motivation by implementing ongoing motivational strategies that are structured, organized, and student oriented.
Such a contention is supported by findings that teachers' attitudes and personalities affect student motivation [23]. In addition, Weller (2005) suggested that internal motivation is more important and longer-lasting than external motivation, which requires constant reinforcement [24]. Another important point to consider pertains to the tremendous adverse effect of clinical requirements on the stress levels of dental students who are in the last 2 years of the academic program; this emphasizes the need for a PDP during these years.

Our study explored student perceptions of the educational environment, by assessing the effect of gender and the change from non-clinical to mostly-clinical education years. This finding could be used to assess and contribute to future academic planning worldwide. Accordingly, necessary measures should be taken to improve those aspects of the educational environment that have been found to require particular attention. However, further studies are needed to address the limitations of this study. These include studying factors that could have affected the study outcome, such as student socio-cultural background, attendance, and academic grade. In addition, future case-control studies that assess the PDP's influence on dental students' perception of the educational environment compared to students in the clinical years that did not include a PDP is recommended. Moreover, a study that assesses students' perception of the educational environment in their final year (6th year), which is mainly clinical, is also recommended.

\section{Conclusion}

Dental students' perceptions of the educational environment at KAUFD support the findings of national and international studies. Students in the preclinical dental 
academic year (third-year) obtained the highest DREEM score, when compared to those in the clinical academic years. Therefore, a PDP and good support systems must be emphasized for clinical-year students.

\section{Supplementary information}

Supplementary information accompanies this paper at https://doi.org/10. 1186/s12909-020-02165-7.

Additional file 1 Supplementary Table 1: Bonferroni correction for statistical differences between the DREEM items scores distributed according to year of graduation.

\section{Abbreviations}

KAUFD: King Abdulaziz university faculty of dentistry; DREEM: Dundee ready education environment measure; PDP: personal development program

\section{Acknowledgements}

Not applicable.

\section{Authors' contributions}

HS designed, analyzed, and contributed in collecting the data, interpreting the results, and writing the manuscript. $\mathrm{HB}$ contributed in writing the manuscript and interpreting the data. $\mathrm{HA}$ and $\mathrm{MB}$ contributed in collecting and organizing the data. All authors read and approved the final manuscript.

\section{Funding}

Not applicable.

\section{Availability of data and materials}

Data generated or analyzed during this study are included in this published article.

\section{Ethics approval and consent to participate}

Written informed consent was obtained from all participants (All participants were above 16 years of age). An ethical approval was obtained from the ethics committee of KAUFD (047-03-18) for this study.

\section{Consent for publication}

Written informed consent for publication of the students' educational environmental perception details was obtained from the students and approved by the KAUFD ethical committee. A copy of the consent form is available for review by the Editor of this journal if requested from the corresponding author.

\section{Competing interests}

There are no competing interests.

\section{Author details}

${ }^{1}$ Department of Pediatric Dentistry, King Abdulaziz University, Jeddah, Saud Arabia. ${ }^{2}$ King Abdulaziz University Faculty of Dentistry, Jeddah, Saudi Arabia.

Received: 11 July 2019 Accepted: 21 July 2020

Published online: 29 July 2020

\section{References}

1. Genn J. AMEE medical education guide no. 23 (part 1): curriculum, environment, climate, quality and change in medical education-a unifying perspective. Med Teach. 2001;23(4):337-44.

2. Dent J, Harden R. A practical guide for medical teachers. Edinburgh: Elsevier Churchill Livingstone; 2009. https://www.worldcat.org/title/practical-guidefor-medical-teachers/oclc/276668853.

3. Newble DI, Cannon RA: A handbook for medical teachers: springer science \& business media; 2001.

4. Roff S, McAleer S, Harden RM, Al-Qahtani M, Ahmed AU, Deza H, Groenen $\mathrm{G}$, Primparyon P. Development and validation of the Dundee ready education environment measure (DREEM). Med Teach. 1997;19(4):295-9.

5. Dentistry KAUfo: Strategic plan. In.; 2017.
6. Al Kuwaiti A. Critical appraisal of the Saudi dental curriculum and a proposal to improve the teaching and learning methods adopted at Saudi dental colleges. Int Med Publishing Group; PHCJ. 2014;9:11-8.

7. McAleer S, Roff S. A practical guide to using the Dundee ready education environment measure (DREEM). AMEE Med Educ Guide. 2001;23(5):29-33.

8. Dunne F, McAleer S, Roff S. Assessment of the undergraduate medical education environment in a large UK medical school. Health Educ J. 2006; 65(2):149-58.

9. Miles S, Swift L, Leinster SJ. The Dundee ready education environment measure (DREEM): a review of its adoption and use. Med Teach. 2012;34(9): e620-34.

10. Al-Mohaimeed A. Perceptions of the educational environment of a new medical school, Saudi Arabia. Int J Health Sci. 2013;7(2):150.

11. Al Sheikh MH. Educational environment measurement, how is it affected by educational strategy in a Saudi medical school? A multivariate analysis. J Taibah Univ Med Sci. 2014;9(2):115-22.

12. Al-Saleh S, Al-Madi EM, AlMufleh B, Al-Degheishem A-H. Educational environment as perceived by dental students at King Saud University. Saudi Dent J. 2018;30(3):240-9.

13. Khan SQ, Al-Shahrani M, Khabeer A, Farooqi FA, Alshamrani A, Alabduljabbar AM, Bahamdan AS, Alqathani MA. Medical students' perception of their educational environment at imam Abdulrahman bin Faisal University, Kingdom of Saudi Arabia. J Fam Community Med. 2019;26(1):45.

14. Aghamolaei T, Fazel I. Medical students' perceptions of the educational environment at an Iranian medical Sciences University. BMC Med Educ. 2010;10(1):87.

15. Bassaw B, Roff S, McAleer S, Roopnarinesingh S, De Lisle J, Teelucksingh S, Gopaul S. Students' perspectives on the educational environment, Faculty of Medical Sciences, Trinidad. Med Teach. 2003;25(5):522-6.

16. Ostapczuk M, Hugger A, De Bruin J, Ritz-Timme S, Rotthoff T. DREEM on, dentists! Students' perceptions of the educational environment in a German dental school as measured by the Dundee ready education environment measure. Eur J Dent Educ. 2012;16(2):67-77.

17. Roff $S$, McAleer S, Ifere O, Bhattacharya S. A global diagnostic tool for measuring educational environment: comparing Nigeria and Nepal. Med Teach. 2001;23(4):378-82.

18. Ali K, McHarg J, Kay E, Moles D, Tredwin C, Coombes L, Heffernan E. Academic environment in a newly established dental school with an enquiry-based curriculum: perceptions of students from the inaugural cohorts. Eur J Dent Educ. 2012;16(2):102-9.

19. Palés J, Gual A, Escaneroi J, Tomás I, de Castro FR, Elorudy M, Virumbrales M, Rodríguez G, Arce V. Educational climate perception by preclinical and clinical medical students in five Spanish medical schools. Int J Med Educ. 2015:6:65.

20. Demiroren M, Palaoglu O, Kemahli S, Ozyurda F, Ayhan I. Perceptions of students in different phases of Medicai education of educational environment: Ankara University Facuity of medicine. Med Educ Online. 2008 13(1):4477.

21. Mittal R, Mahajan R, Mittal N. Foundation programme: a student's perspective. Int J Appl Basic Med Res. 2013;3(1):52

22. Nebhinani N, Ghatak S, Singh K, Misra S, Bhagat OL, Garg R. Effect of personality development program for medical and nursing students: a pilot study. Ann Natl Acad Med Sci (India). 2015;51(1 \& 2):13-9.

23. Shadlyn, Robyn: Motivation. In., 5 February 2011 edn; 2004.

24. Weller, Matthew: General Principles of Motivation. In., 20 February 2011 edn: Los Angeles Journal; 2005.

\section{Publisher's Note}

Springer Nature remains neutral with regard to jurisdictional claims in published maps and institutional affiliations. 